\title{
5 Research Square

\section{Vulnerabilities of High-Security Forensic Psychiatry Residences in Italy during the COVID-19 pandemic: a qualitative survey and a proposal for recovery and prevention}

\section{Andrea Pozza ( $\square$ andrea.pozza@unisi.it )}

University of Siena

\section{Fabio Ferretti}

University of Siena

Fulvio Carabellese

University of Siena

Giacomo Gualtieri

University of Siena

Alessandra Masti

University of Siena

Felice Carabellese

University of Bari "Aldo Moro"

Anna Coluccia

University of Siena

\section{Research Article}

Keywords: COVID-19, vulnerability factors, pandemic, forensic psychiatry, recovery, qualitative survey.

Posted Date: September 29th, 2020

DOI: https://doi.org/10.21203/rs.3.rs-83482/v1

License: (a) (i) This work is licensed under a Creative Commons Attribution 4.0 International License. Read Full License 


\section{Abstract}

Italy is the first European country severely hit by the COVID-19 pandemic after the outbreak in Wuhan. In our healthcare context, the closing of forensic psychiatric hospitals in 2014 completed the long transition from a containment approach for offenders with severe mental disorders to a model oriented to recovery and community reintegration. High-Security Forensic Psychiatry Residences (REMS) were implemented by regional health authorities. Within these settings, inpatients with severe psychiatric disorders who committed a criminal offense, without criminal responsibility but still considered socially dangerous, are held, and treated. The current COVID-19 emergency represents a severe stressful event which has a potentially negative impact on the recovery process of the inpatients and on the work-related health of the psychiatric professionals.

The present paper consists of two sections. In the first one, we discuss the organizational and clinical issues emerged in the forensic psychiatry system in Italy. We identify vulnerable groups and processes and suggest long-term prevention strategies at both clinical and organizational levels. Within the second section, we present the findings of a qualitative exploratory survey conducted in four different REMS immediately after the stop of the lockdown. Based on a qualitative approach, this survey explored the COVID-19 effects on mental health of REMS inpatients and healthcare workers. Thematic analysis showed five key themes: "Intervention and prevention strategies during the lockdown decision making";

"Inpatients' reactions to the pandemic"; "Psychological reactions of the healthcare staff"; "Recovery process and relations with community mental health services"; "Lessons for the future learned from the COVID-19".

\section{Introduction}

\section{High-Security Forensic Psychiatry Residences in Italy: a recovery-oriented model}

In Italy, the closing of six forensic psychiatric hospitals in 2014 completed the long transition from a containment approach for offenders with mental disorders to a psychiatric model oriented to recovery and community reintegration (Carabellese and Felthous 2016). From 2014 to 2017, 20-bed, High-Security Forensic Psychiatry Residences (REMS: "Residenze per le Misure di Sicurezza") were built and implemented by regional health authorities. Within these settings inpatients with psychiatric disorders who committed a criminal offense, without criminal responsibility but still considered socially dangerous, are held and treated. The healthcare professionals encourage inpatients to participate in a recoveryoriented rehabilitation project, in order to return them to community services as soon as possible (Catanesi et al. 2019; Zanalda et al. 2020).

High-Security Forensic Psychiatry Residences have been conceived as high-cost, low-volume services which pose significant restrictions on individuals. It is therefore very important that the practice of such services is consistent with the highest standards and is based on the best quality evidence (Barbui and 
Saraceno 2015; Völlm et al. 2018). Indeed, the Law n 24 made on $8^{\text {th }}$ March 2017 (the so-called "Gelli Law') stated that specific evidence-based guidelines should be applied to avoid incurring professional responsibility charges since inpatients held in REMS who are often at risk of self-harm or violent acts towards the forensic psychiatric staff (Carabellese et al. 2017; Coluccia et al. 2017).

\section{The impact of the COVID-19 pandemic in Italy}

Italy is the first European country severely hit by the COVID-19 pandemic after the outbreak in Wuhan, as the first positive patient was diagnosed on February 18 in Codogno, a small northern city (Grasselli et al. 2020). Due to a number of issues including the reluctance of the politicians to adopt timely containment measures and the controversial information provided by social media on the pandemic, coronavirus circulated throughout the country for some weeks. On March 11, the government decided to introduce the lockdown to the whole national territory. The progression of the pandemic however, increased for the mounting demands of hospitalization.

The COVID-19 outbreak in Italy had a strong impact also on the forensic psychiatry system. The current emergency should be regarded as a severe stressful event which has a potentially negative impact on the recovery process of the inpatients and on the work-related health of the psychiatric professionals.

The present paper consists of two sections. In the first one, we discuss the problematic organizational and clinical issues emerged in the forensic psychiatry system in Italy, and the key directions for the future; within the second section, we present the findings of a qualitative survey conducted in four different REMS immediately after the stop of the lockdown. Based on a qualitative approach, this survey aimed to explore the COVID-19 effects on mental health of REMS inpatients and healthcare workers, detect potential organizational and clinical issues and identify future directions.

\section{Identifying vulnerable groups and preventing long-term outcomes in forensic psychiatry settings during the COVID-19 pandemic}

According to the Inter-Agency Standing Committee guidelines for "Mental Health and Psychosocial Support" during emergency situations (Bragin 2020), a wide variety of strategies should be used, such as identifying vulnerable groups, preventing long-term negative outcomes, adopting participatory approaches, and building on existing resources. Addressing the organizational gaps and the long-term clinical outcomes in forensic psychiatry settings is one of the key challenges for public healthcare system in Italy during the COVID-19 pandemic.

In our forensic psychiatry system, a first problematic aspect is given by the lack of scientific evidence for psycho-rehabilitative treatments in these special settings for reducing violent/aggressive behaviours as a direct outcome (Trestman 2017). European Psychiatric Association's working group on forensic 
psychiatry (Völlm et al. 2018) provided a guidance paper of best evidence and practice on psychopharmacological and psychological interventions for inpatient offenders with mental disorders. However, in Italy little attention is dedicated to guidelines and poor evidence is still available regarding the best strategies to be implemented in the management of healthcare emergencies within forensic psychiatry settings (D'Agostino et al. 2020; Zuffanieri and Zanalda 2020).

The use of an evidence-based approach is strategic since this can allow the most reliable and effective assessment and treatment procedures to be adopted, particularly during emergency situations like the present one. Despite some preliminary effectiveness studies have been conducted in the Italian context (e.g., Bianchini et al. 2019), more research and clinical work should be done to evaluate and adapt the psycho-rehabilitative programs to our context, since the attention given by forensic psychiatry professionals to evidence-based literature is still limited. The lack of evidence-based programs makes the REMS high-risk settings during the COVID-19 pandemic, as the spread of controversial information on the pandemic might have a strong impact on the inpatients' distress and increase the risk for violent acts (Campbell 2020).

As compared with the professionals working in other criminal law systems (e.g., Messina et al. 2019), Italian forensic psychiatry professionals have still poor skills in the use of guidelines and evidence-based scales/interventions. Indeed, specialist residency university programs are not sufficiently focused on these topics. We believe that the development of $\mathrm{PhD}$ courses on forensic medical sciences focused on evidence-based assessment and treatment in forensic psychiatry settings may increase the expertise of the professionals working in these contexts.

An evidence-based approach may also be particularly useful in identifying at-risk groups of offenders. The use of reliable instruments of the potentiality for outbreaks of violence is crucial in High-Security Forensic Psychiatry Residences since these inpatients have been identified as a danger to society, and this risk may be higher during emergency situations. They have more, and often different, risk factors that need for a timely risk management in order to prevent violent acts against staff or other inpatients. Thus, the use of evidence-based scales aimed to detect at-risk inpatients should be implemented in Italy. For example, the Historical-Clinical-Risk Management-20, Version 3 (HCR-20V3; Douglas et al. 2014) might be useful in the violence risk assessment based on historical and dynamic factors. This tool has been recently validated by a group of Italian forensic psychiatrists and psychologists (Caretti et al. 2019). The evaluation of dangerousness might also include the assessment of the stages of violence risk, for example through the DUNDRUM (Davoren et al. 2012), an instrument aimed to support professional judgment with respect to the need for therapeutic security, treatment completion, and recovery.

In the Italian context, the COVID-19 pandemic might also exacerbate psychopathological symptoms in forensic psychiatric inpatients held in REMS. Specific groups of offenders should be considered at-risk of an increased psychological distress during the coronavirus outbreak. For example, it has been demonstrated that child molesters have exaggerated disgust reactions to the thought of coming into physical contact with adults, but not children. Indeed, child molesters, in comparison to non-offenders 
and violent offenders, showed excessive concern at the thought of being touched on the lips or tongue with a re-sterilized surgical swab (Mitchell et al. 2012). Other data suggest that child molesters show clinically relevant obsessive-compulsive symptoms (Marshall et al. 2012). Therefore, reliable measures of contamination fears should be introduced to identify vulnerable groups. For example, the Obsessive Compulsive Inventory-Revised (OCl-R; Huppert et al. 2007) is a short self-report questionnaire, easy to administer periodically; it might be used to monitor the levels of contamination fears, a psychological subjective experience frequently occurring during a pandemic like the present one (Cisler et al. 2007; Pozza et al. 2020). In addition, forensic psychiatry professionals should be assessed with respect to the risk of contamination fear, as previous evidence showed that this professional group typically experiences disgust reactions towards the offenders (Jacob and Holmes, 2011), and disgust propensity has been shown to prospectively predict the onset of clinically relevant contamination symptoms during stressful circumstances (Olatunji 2010).

Another important consequence of the COVID-19 pandemic may be the development of traumatic symptoms in the offenders and professionals, particularly in the contexts where some infected individuals are identified. The assessment of traumatic symptoms may be a key aspect to be monitored in offenders with psychiatric disorders (Allely and Allely, 2020). Indeed, this population may either have a history of previous traumatic events (e.g., childhood traumatic events), or develop offence-related posttraumatic stress disorder after the commission of the crime or have poor emotion regulation skills and impulsivity (Crisford et al. 2008; Lewis and Ireland, 2019; Wilson et al. 2013).

With respect to the vulnerability to traumatic features, clinical and gender differences should be taken into account, with inpatients having borderline personality disorder and women respectively being more predisposed to develop such type of symptoms (Bohle and de Vogel, 2017; Carabellese et al. 2018; Carabellese et al. 2019a, 2019b; Carabellese et al. 2020; Sinai et al. 2018).

Some psychotherapeutic approaches have been empirically validated through well-conducted trials for high-risk groups in forensic psychiatric systems. However, as previously noted, there is still poor attention to evidence-based treatments within forensic settings and no guideline papers do exist in Italy. This seems to be particularly relevant since the Italian forensic psychiatry system is less prepared for mental health prevention or early intervention in at-risk situations. Little effort is dedicated to the implementation of strategies aimed to strengthen the psychological resources of the forensic psychiatric inpatients and healthcare staff working in these settings. Given the high risk for the development of post-traumatic symptoms during the pandemic (Huang et al. 2020), trauma-informed care approaches should be added to the interventions routinely delivered in Italian forensic psychiatry settings (Lewis and Ireland, 2019). A number of empirically validated psychotherapeutic approaches have been developed to reduce traumarelated symptoms such as Eyes Movement Desensitization Reprocessing (EMDR), or to prevent them such as mindfulness-based protocols, both for the offenders and the psychiatric staff (Every-Palmer et al. 2019; Howell et al. 2010). 
In addition to the inpatients, another vulnerable group is the healthcare staff working in High-Security Forensic Psychiatry Residences, that has to cope with a number of sources of psychological distress, including the fear to get contaminated and to contaminate their families and the inpatients, and higher levels of burnout. In the literature, various instruments have been developed to assess distorted thinking styles, such as perfectionistic beliefs or the tendency to perceive an inflated sense of responsibility for harming/infecting other people such as the Obsessive Beliefs Questionnaire-44 (Steketee et al. 2003). These cognitive factors can maintain high levels of work-related stress and should be regarded as early warning signs of severe burnout during emergency situations (Jenkins and Elliott 2004). Cognitive behavioural therapy techniques have been demonstrated to be effective for reducing such maladaptive beliefs predisposing mental health professionals to high levels of burnout (Ewers et al. 2002).

The assessment of social climate and burnout levels amongst the psychiatric professionals should be improved by adding the periodic administration of specific scales during the pandemic. For example, Tonkin (2016) conducted a systematic review of measures developed to assess social climate in forensic psychiatric wards. The results showed that the Essen Climate Evaluation Schema (EssenCES; Schalast and Laan 2017) was associated with the most robust factorial validity, the best reliability evidence, and the most satisfactory convergent validity performance. This measure is aimed at covering three main elements of social climate in forensic psychiatric environments, including residents' cohesion, therapeutic hold, and experienced safety (Schalast and Laaan, 2017).

We also suggest the use of measures of compassion-focused environments which may be helpful in improving the recovery-oriented approach of the rehabilitation programs (Veale et al. 2015). While the assessment of distress-related measures is a key element, we believe that a broader attention to positive outcomes should be implemented to more closely monitor the recovery process, such as psychological wellbeing, quality of life, self-efficacy, health literacy, and coping (Logan et al. 2019; Ryff and Keyes 1995). All these resources might have an important role during the discharge, particularly at the coronavirus time. Mindfulness-based interventions might be helpful in strengthening these resources, reducing the distress in the post-emergency phase and preventing a range of long-term psychopathological outcomes in both the offenders and healthcare professionals, as suggested by previous reviews and well-conducted randomized controlled trials (Banks et al. 2015; Burton et al. 2017; Irving et al. 2009).

Another issue regarding the psycho-rehabilitation programs is the implementation of social distancing measures and quarantine which may slow the recovery process of the inpatients. In addition, due to social distancing measures, group therapy sessions are not possible, and this can reduce the improvement on key therapeutic processes such as the development of insight, empathetic skills, and emotion regulation strategies (Zhou et al. 2018). The problematic effects of the quarantine may also concern the implementation of occupational therapy pathways aimed to foster community reintegration (Perilli et al. 2018). 
Thus, the challenge of the COVID-19 pandemic calls for the implementation of telepsychiatry tools as integrated modality for the management of the inpatients in forensic settings. Despite the strategic relevance of telemedicine has increasingly received the attention by policymakers in Italy (Di Lenarda et al. 2017), the usefulness of remote consultations is still under-estimated in forensic psychiatry.

Telepsychiatry tools may be effectively applied to the administration of assessment measures and delivery of psychotherapy. As shown elsewhere (Chakrabarty 2015; Shore et al. 2007), such tools may increase the self-management skills of less severe inpatients during the advanced phase of the recovery process. In addition, they may be helpful for staff meetings, thus in improving the staff communication during the pandemic when in-presence meetings may not always be possible.

Finally, the COVID-19 pandemic has also raised the problem of lack of psychological support for the relatives of the offenders since in our context poor attention is given to evidence-based prevention programs aimed at reinforcing the skills of the relatives in the management of the offender after the discharge. Therefore, in order to facilitate the recovery process, the emergence of psychopathological symptoms and other negative outcomes should be carefully monitored in the relatives of the offenders during the pandemic by assessing key variables such as the levels of burden, anger, post-traumatic symptoms, expressed emotions and health literacy, often associated with a higher risk of relapse (Bustnay 2020).

\section{Covid-19 Effects In Forensic Psychiatry Settings: A Teleconference- based Qualitative Survey}

\section{Background}

Another key aspect concerning the peculiarity of the organization of forensic psychiatry settings in Italy concerns the Law $n^{\circ} 3$ of $18^{\text {th }}$ October 2001 of the fifth Chapter of the Constitution in the section related to the governance of regions. According to this law, each region of Italy has full competence and autonomy for health policy in their territory. In Italy, the protection of health by the state is a right guaranteed by the Constitution. During the pandemic, each region made its own decisions on the security measures to be adopted under the coordination of the Ministry of Health and for all the regional REMS. Lombardy paid the highest price as almost $50 \%$ of all the country's deaths occurred in this region which is the most industrialized one.

Public offices remained closed, including the Courts, during the lockdown in all the country; the Court hearings were postponed or made only if urgent by teleconference for prisoners expiring prison terms. The hospitals guaranteed only urgent care, all outpatient activities were suspended. In the REMS, entrances and discharge were suspended; visits from relatives were also interrupted. Any therapeutic or psycho-social activity usually performed outside of the facility was stopped.

We conducted a teleconference-based qualitative survey based on a semi-structured interview to the mental health professionals of the REMS of four regions located in four different areas of the country: 
Castiglione delle Stiviere (situated in Lombardy in Northern Italy, the most and first severely hit region of our country); Volterra (Tuscany, central Italy); Carovigno REMS (Puglia) and Pisticci REMS (Basilicata), which are located in southern Italy, the least severely hit area of the country.

\section{Methodology}

Study design

The present study was conducted and reported according to the recommendations for qualitative research provided by O'Brien et al. (2014). The study comprised individual semi-structured interviews with the mental health professionals of the four REMS located in four different areas of Italy. All interviews were conducted by a team of three researchers trained in qualitative research methods (texts of the interviews may be requested from the corresponding author). Prior to the conduction of the interviews, the researchers met to share the organization of the interview session. Data were collected in the phase immediately subsequent to the lockdown, from May to June 2020, then they were analysed by the researchers using thematic analysis.

\section{Participants and context}

The mental health professionals of the REMS of four regions located in different areas of Italy were recruited, specifically Castiglione delle Stiviere (situated in Lombardy in Northern Italy, the most and first severely hit region of our country); Volterra (Tuscany, central Italy); Carovigno REMS (Puglia) and Pisticci REMS (Basilicata), which are located in southern Italy, the least severely hit area of the country.

The mental health professionals were recruited through an e-mail invitation from the principal investigator who briefly presented the purposes of the study. Participants were informed that the purpose of the study was to explore the experiences of the directors and colleagues with regard to the COVID-19 effects on the inpatients and healthcare professionals of the REMS.

\section{Data collection and analysis}

Through an interpretative approach, interviews were conducted to explore professionals' experiences with a series of relevant topics concerning REMS inpatients' and healthcare professionals during the lockdown. Interviews took place by video-conference.

A topic guide was developed on the basis of existing literature, through discussions with the research team, who had expertise in forensic psychiatry settings and qualitative methodology, and a pilot interview with REMS professionals. The topic guide was applied flexibly, and revisions were made throughout the 
data collection period to ensure that emerging concepts informed subsequent interviews. Detailed field notes were made during both interviews and coding and used to aid analyses and interpretation. Thematic analysis was drawn on, using an inductive approach to detect, analyse, and report patterns in the data, using a constant comparative method, both between and within transcripts. The process began by audio-recording, then transcribing the interviews verbatim, facilitating data familiarisation through repeated listening. Meaningful units of texts were organised using line-by-line coding, through the prism of the research question.

Codes were organised into groups and named on the basis of analysis. These groups were revised and refined different times until they formed themes. As part of the coding process, codes and themes were regularly presented to the wider research team, who contributed to their refinement and revision. All data were coded and used until the final stage, in which some items deemed irrelevant to the research question were not included.

\section{Results}

Identified codes reflected five key themes: "Intervention and prevention strategies during the lockdown decision making"; "Inpatients' reactions to the pandemic"; "Psychological reactions of the healthcare staff"; "Recovery process and relations with community mental health services"; "Lessons for the future learned from the COVID-19".

\section{Theme 1: "Intervention and prevention strategies during the lockdown"}

The first questions posed to the health professionals of the REMS were that relating to the intervention and prevention plans adopted during the lockdown and any critical issues that emerged following the measures taken. All the REMS adopted a series of protective measures with all inpatients and health professionals including body temperature measurement and protective devices (masks, hand gloves, detergents and sanitizers, disinfection, and cleaning of hospital surfaces). All rooms were sanitized at least twice a day. A space was established to isolate any suspect inpatients. In-person interviews with inpatients were limited to emergencies only. In-person staff meetings were also reduced; teleconferences were made, only if necessary. All the interviewees reported the difficulty in the initial stages of the lockdown in finding personal protective devices (e.g. masks and gloves, detergents, also buffers) aimed at containing the spread of the infection.

Only in the REMS of Castiglione delle Stiviere in Lombardy, about $20 \%$ of health professionals tested positive for Covid-19 and were removed from the REMS. Some inpatients who entered before the lockdown also tested positive; they were isolated but none of them needed hospitalization.

The director of a REMS also tested positive for COVID 19 and was dismissed but, fortunately, none of the health professionals tested positive in this REMS. 


\section{Theme 2: "Inpatients' reactions to the pandemic"}

The subsequent questions aimed at ascertaining how the REMS inpatients had reacted to the restrictions imposed by the lockdown, their cognitive, emotional, and behavioural responses, any problems that emerged during this phase. Surprisingly, all inpatients reacted adequately despite the severe limitations received, and despite the fact that the clinical in-person interviews had been almost completely suspended. Interestingly, there were no psychopathological decompensations during this period: there were no psychotic breakdowns or hospitalizations, and the incidence of psychiatric hospitalizations during the lockdown were much lower than during the previous months and the same period of 2019. As highlighted by all the interviewees, the possibility to call their relatives by phone was probably a protective factor for the inpatients against the onset of psychopathological decompensation.

Theme 3: "Psychological reactions of the healthcare staff"

After an initial phase of staff's difficulty during which some of the workers showed denial reactions or resistance to adopt the protection procedures, the staff of all REMS responded adequately. Protective devices were provided for all health professionals.

The director of a REMS also tested COVID-19 positive and for this the health professionals interviewed reported initial concerns and anxious feelings. The REMS of Castiglione delle Stiviere implemented a psychological in-person support group for the whole staff.

Theme 4: "Recovery process and relations with community mental health services"

The subsequent questions aimed at ascertaining how the staff managed the recovery process and relations with other community mental health services during the lockdown and the subsequent phase. In this regard, all directors reported the complete suspension of external rehabilitation activities, relations with external social agencies, home visits, health check-ups. All interviewees agreed that these inevitable restrictions had a negative impact for the recovery process and discharge from the REMS.

\section{Theme 5: "Lessons for the future learned from the COVID-19"}

All interviewees agreed that a greater attention to hygiene was a positive effect of the COVID-19 that highlighted the importance of personal hygiene in forensic psychiatry settings and a closer monitoring of the inpatients. In contrast, the emergency situation showed that the strategic use of telemedicine is still 
under-recognized and under-utilized in Italian forensic psychiatry settings where the therapeutic relationship with the patient is very important.

\section{General Discussion And Concluding Remarks}

Italy is the first European country severely hit by the COVID-19 pandemic after the outbreak in Wuhan. In our healthcare context, the closing of forensic psychiatric hospitals in 2014 completed the long transition from a containment approach for offenders with severe psychiatric disorders to a model oriented to recovery and community reintegration. The function of REMS, a new form of recovery-oriented forensic psychiatry residences implemented by regional health authorities, is providing psychiatric rehabilitation to individuals with severe psychiatric disorders who committed a criminal offense, still considered socially dangerous. The current pandemic represents a severe healthcare emergency which has a potentially negative impact on the recovery process of the inpatients and on work climate of these settings.

The present paper highlighted the organizational and clinical issues emerged in the forensic psychiatry system in Italy and identified vulnerable groups of inpatients and psychiatric staff that should be more carefully monitored through an organizational approach focused on early detection and prevention. We presented the findings of a qualitative exploratory survey conducted in four different REMS immediately after the stop of the lockdown to assess the psychological impact of COVID-19 pandemic on the mental health of REMS inpatients and healthcare workers. Specifically we focused on the strategies adopted by the REMS during the lockdown to prevent COVID-19 transmission, the psychological reactions to the pandemic reported by the inpatients, the psychological reactions of the healthcare staff, potential changes in the relations with community mental health services during the lockdown, and the lessons for the future learned from the COVID-19. The strength of this is that this is the first empirical contribution which explored the psychological impact of the pandemic on forensic psychiatry settings in Italy. Our findings suggest the implementation of evidence-based screening and prevention approaches which are still neglected in our country.

It is very interesting that none of inpatients reported acute psychopathological decompensations during the lockdown period (i.e., hospitalization or psychotic breakdown). This outcome should lead health policies to focus on the implementation of strategies aimed at preventing the occurrence of negative mental health outcomes in both inpatients and healthcare professionals at long-term.

While the use of in-depth interviews enabled us to identify key themes with respect to this topic and provide some preliminary data, this methodological approach represents a limitation that should be overcome by quantitative research and longitudinal designs used in much larger samples.

Future directions include the development of standardised early detection and prevention strategies based on the available evidence to identify vulnerable inpatients and healthcare workers and timely implement preventive measures. One of the most relevant limits of our healthcare system regards the lack of importance given to telemedicine-based practices which should be more carefully considered for the future. 


\section{Declarations}

Participants consented to participate and publish the results

The authors of the paper declare no conflict of interests

\section{References}

Allely, C. S., Allely, B. (2020). Post Traumatic Stress Disorder in incarcerated populations: current clinical considerations and recommendations. Journal of Criminal Psychology.

Banks, K., Newman, E., Saleem, J. (2015). An overview of the research on mindfulness-based interventions for treating symptoms of posttraumatic stress disorder: A systematic review. Journal of Clinical Psychology, 71(10), 935-963.

Barbui, C., Saraceno, B. (2015). Closing forensic psychiatric hospitals in Italy: a new revolution begins?. The British Journal of Psychiatry, 206(6), 445-446.

Bianchini, V., Cofini, V., Curto, M., Lagrotteria, B., Manzi, A., Navari, S., et al. (2019). Dialectical behaviour therapy (DBT) for forensic psychiatric patients: An Italian pilot study. Criminal Behaviour and Mental Health, 29(2), 122-130.

Bohle, A., Vogel, V. (2017). Gender differences in victimization and the relation to personality disorders in forensic psychiatry. Journal of Aggression, Maltreatment \& Trauma, 26(4), 411-429.

Bragin, M. S. (2020). Bragin, M. S. (2020). Inter-Agency Guidelines for Psychosocial Intervention in Emergencies. In Encyclopedia of Social Work. Retrieved on 08/08/2020 from https://oxfordre.com/socialwork/view/10.1093/acrefore/9780199975839.001.0001/acrefore9780199975839-e-1256

Burton, A., Burgess, C., Dean, S., Koutsopoulou, G. Z., Hugh-Jones, S. (2017). How effective are mindfulness-based interventions for reducing stress among healthcare professionals? A systematic review and meta-analysis. Stress and Health, 33(1), 3-13.

Bustnay, T. G. (2020). Group intervention with parents of juvenile sex offenders. Journal of child Sexual Abuse, 29(3), 278-294.

Campbell, A. M. (2020). An increasing risk of family violence during the Covid-19 pandemic:

Strengthening community collaborations to save lives. Forensic Science International: Reports, 100089.

Carabellese, F., Felthous, A. R. (2016). Closing Italian forensic psychiatry hospitals in favor of treating insanity acquittees in the community. Behavioral Sciences \& the Law, 34(2-3), 444-459. 
Carabellese F., Urbano M., Coluccia A., Gualtieri G. (2017). Workers safety in public psychiatric services: Problems, laws and protections. La Clinica Terapeutica, 168, 271-279.

Carabellese F., Felthous A., Rossetto I., La Tegola D., Franconi F., Catanesi R. (2018). Female Residents with Psychopathy in a High-Security Italian Hospital. The Journal of the American Academy of Psychiatry and the Law, 46, 171-178.

Carabellese, F., Felthous, A. R., Montalbo, D., La Tegola, D., Rossetto, I., Franconi, F., Catanesi, R. (2019a). Psychopathy and Female Gender: Phenotypic Expression and Comorbidity: A Study Comparing a Sample of Women Hospitalized in Italy's Maximum Security Facility with Women who were Criminally Sentenced and Imprisoned. Journal of Forensic Sciences, 64(5), 1438-1443.

Carabellese, F., Felthous, A.R., Mandarelli, G., Montalbo, D., La Tegola, D., Rossetto, I., Franconi, F., and Catanesi, R. (2019b). Psychopathy in Italian female murderers. Behavioral Sciences and the Law, 37(5), 602-613. DOI: $10.1002 / \mathrm{bsl} .2430$

Carabellese, F., Felthous, A. R., La Tegola, D., Rossetto, I., Franconi, F., Lucchini, G., Catanesi, R. (2020). Female psychopathy: a descriptive national study of socially dangerous female NGRI offenders. International Journal of Law and Psychiatry, 68.

Caretti, V., Carpentieri, R., Caprì, C., Schimmenti, A. (2019). Attendibilità, validità e proprietà psicometriche della versione italiana dell" HCR-20v3. Firenze: Hogrefe F, editor.

Catanesi, R., Mandarelli, G., Ferracuti, S., Valerio, A., Carabellese, F. (2019). The new Italian residential forensic psychiatric system (REMS). A one-year population study. Italian Journal of Criminology, 13, 7-23.

Chakrabarti, S. (2015). Usefulness of telepsychiatry: A critical evaluation of videoconferencing-based approaches. World Journal of Psychiatry, 5(3), 286.

Cisler, J. M., Reardon, J. M., Williams, N. L., Lohr, J. M. (2007). Anxiety sensitivity and disgust sensitivity interact to predict contamination fears. Personality and Individual Differences, 42(6), 935-946.

Coluccia, A., Nucci, G., Cuomo, A., Benvenuti, M., Carabellese, F., Fagiolini, A., Gabbrielli M. (2017). Duties and liabilities for psychiatrists. Rivista di Psichiatria, 52(6), 226-235.

Crisford, H., Dare, H., Evangeli, M. (2008). Offence-related posttraumatic stress disorder (PTSD) symptomatology and guilt in mentally disordered violent and sexual offenders. The Journal of Forensic Psychiatry \& Psychology, 19(1), 86-107

D'Agostino, A., Demartini, B., Cavallotti, S., Gambini, O. (2020). Mental health services in Italy during the COVID-19 outbreak. The Lancet Psychiatry, 7(5), 385-387.

Davoren, M., O'Dwyer, S., Abidin, Z., Naughton, L., Gibbons, O., Doyle, E., et al. (2012). Prospective inpatient cohort study of moves between levels of therapeutic security: the DUNDRUM-1 triage security, 
DUNDRUM-3 programme completion and DUNDRUM-4 recovery scales and the HCR-20. BMC Psychiatry, 12(1), 80.

Di Lenarda, A., Casolo, G., Gulizia, M. M., Aspromonte, N., Scalvini, S., Mortara, A., et al. (2017). The future of telemedicine for the management of heart failure patients: a Consensus Document of the Italian Association of Hospital Cardiologists (ANMCO), the Italian Society of Cardiology (SIC) and the Italian Society for Telemedicine and eHealth (Digital SIT). European Heart Journal Supplements, 19(suppl_D), D113-D129.

Douglas, K. S., Hart, S. D., Webster, C. D., Belfrage, H., Guy, L. S., Wilson, C. M. (2014). Historical-clinicalrisk management-20, version 3 (HCR-20V3): development and overview. International Journal of Forensic Mental Health, 13(2), 93-108.

Every-Palmer, S., Flewett, T., Dean, S., Hansby, O., Colman, A., Weatherall, M., et al. (2019). Eye movement desensitization and reprocessing (EMDR) therapy for posttraumatic stress disorder in adults with serious mental illness within forensic and rehabilitation services: A study protocol for a randomized controlled trial. Trials, 20(1), 642.

Ewers, P., Bradshaw, T., McGovern, J., Ewers, B. (2002). Does training in psychosocial interventions reduce burnout rates in forensic nurses?. Journal of Advanced Nursing, 37(5), 470-476.

Grasselli, G., Pesenti, A., Cecconi, M. (2020). Critical care utilization for the COVID-19 outbreak in Lombardy, Italy: early experience and forecast during an emergency response. JAMA, 323(16), 15451546.

Howell, A. J., Digdon, N. L., Buro, K. (2010). Mindfulness predicts sleep-related self-regulation and wellbeing. Personality and Individual Differences, 48(4), 419-424.

Huang, J. Z., Han, M. F., Luo, T. D., Ren, A. K., \& Zhou, X. P. (2020). Mental health survey of 230 medical staff in a tertiary infectious disease hospital for COVID-19. Zhonghua lao dong wei sheng zhi ye bing za zhi= Zhonghua laodong weisheng zhiyebing zazhi= Chinese journal of industrial hygiene and occupational diseases, 38, E001-E001.

Huppert, J. D., Walther, M. R., Hajcak, G., Yadin, E., Foa, E. B., Simpson, H. B., et al. (2007). The OCI-R: validation of the subscales in a clinical sample. Journal of Anxiety Disorders, 21(3), 394-406.

Irving, J. A., Dobkin, P. L., Park, J. (2009). Cultivating mindfulness in health care professionals: A review of empirical studies of mindfulness-based stress reduction (MBSR). Complementary Therapies in Clinical Practice, 15(2), 61-66.

Jacob, J. D., Holmes, D. (2011). Working under threat: Fear and nurse-patient interactions in a forensic psychiatric setting. Journal of Forensic Nursing, 7(2), 68-77. 
Jenkins, R., Elliott, P. (2004). Stressors, burnout and social support: nurses in acute mental health settings. Journal of Advanced Nursing, 48(6), 622-631.

Lewis, M., Ireland, J. L. (2019). Understanding motives for aggression in forensic psychiatric patients: A preliminary study. The Journal of Forensic Psychiatry \& Psychology, 30(3), 496-509.

Logan, R. A., Wong, W. F., Villaire, M., Daus, G., Parnell, T. A., Willis, E., Paasche-Orlow, M. K. (2015). Health literacy: A necessary element for achieving health equity. NAM Perspectives.

Marshall, L. E., O’Brien, M. D., Marshall, W. L., Booth, B., Davis, A. (2012). Obsessive-compulsive disorder, social phobia, and loneliness in incarcerated Internet child pornography offenders. Sexual Addiction \& Compulsivity, 19(1-2), 41-52.

Messina, E., Ferracuti, S., Nicolò, G., Ruggeri, M., Kooijmans, T., Meynen, G. (2019). Forensic psychiatric evaluations of defendants: Italy and the Netherlands compared. International Journal of Law and Psychiatry, 66, 101473.

Mitchell, I. J., Keylock, H., Campbell, N., Beech, A. R., Kogan, D. (2012). Do child molesters show abnormal disgust and fear of contamination reactions?. Psychiatry, Psychology and Law, 19(2), 282-294.

O’Brien, B. C., Harris, I. B., Beckman, T. J., Reed, D. A., Cook, D. A. (2014). Standards for reporting qualitative research: a synthesis of recommendations. Academic Medicine, 89(9), 1245-1251.

Perilli, V., Stasolla, F., Maselli, S., Morelli, I. (2018). Occupational therapy and social skills training for enhancing constructive engagement of patients with schizophrenia: A review. Clinical Research in Psychology, 1(1), 1-7.

Pozza, A., Mucci, F., Marazziti, D. (2020). Risk for pathological contamination fears at coronavirus time: Proposal of early intervention and prevention strategies. Clinical Neuropsychiatry, 17(2), 100-102.

Ryff, C. D., Keyes, C. L. M. (1995). The structure of psychological well-being revisited. Journal of Personality and Social Psychology, 69(4), 719-727.

Schalast, N., \& Laan, J. M. (2017). Measuring social climate in German prisons using the Essen Climate Evaluation Schema. The Prison Journal, 97(2), 166-180.

Shore, J. H., Hilty, D. M., Yellowlees, P. (2007). Emergency management guidelines for telepsychiatry. General Hospital Psychiatry, 29(3), 199-206.

Sinai, C., Hirvikoski, T., Wiklander, M., Nordström, A. L., Nordström, P., Nilsonne, Å., et al. (2018). Exposure to interpersonal violence and risk of post-traumatic stress disorder among women with borderline personality disorder. Psychiatry Research, 262, 311-315. 
Steketee, G., Frost, R., Bhar, S., Bouvard, M., Calamari, J., Carmin, C., et al. (2003). Psychometric validation of the obsessive beliefs questionnaire and the interpretation of intrusions inventory: Part I. Behaviour Research and Therapy, 41(8), 863-878.

Tonkin, M. (2016). A review of questionnaire measures for assessing the social climate in prisons and forensic psychiatric hospitals. International Journal of Offender Therapy and Comparative Criminology, 60(12), 1376-1405.

Trestman, R. L. (2017). Treating Aggression in Forensic Psychiatric Settings. The journal of the American Academy of Psychiatry and the Law, 45(1), 40-43.

Veale, D., Gilbert, P., Wheatley, J., Naismith, I. (2015). A new therapeutic community: Development of a compassion-focussed and contextual behavioural environment. Clinical Psychology \& Psychotherapy, 22(4), 285-303.

Völlm, B. A., Clarke, M., Herrando, V. T., Seppänen, A. O., Gosek, P., Heitzman, J., et al. (2018). European Psychiatric Association (EPA) guidance on forensic psychiatry: Evidence based assessment and treatment of mentally disordered offenders. European Psychiatry, 51, 58-73.

Wilson, C. M., Desmarais, S. L., Nicholls, T. L., Hart, S. D., Brink, J. (2013). Predictive validity of dynamic factors: Assessing violence risk in forensic psychiatric inpatients. Law and Human Behavior, 37(6), 377.

Zanalda, E., De Cori, D., Ala, G., Sodano, A. J., Zuffranieri, M. (2020). Violent Behavior in Forensic Residential Facilities: The Italian Experience After the Closure of Forensic Psychiatric Hospitals. In B. Carpiniello, A. Vita, C. Mencacci (eds.), Violence and Mental Disorders (pp. 211-227). Springer: Cham.

Zhou, Y. Q., Gan, D. Z. Q., Hoo, E. C. C., Chong, D., Chu, C. M. (2018). Evaluating the violence prevention program: group and individual changes in aggression, anger, self-control, and empathy. The Journal of Forensic Psychiatry \& Psychology, 29(2), 265-287.

Zuffranieri, M., Zanalda, E. (2020). The Forensic Psychiatry in the time of Coronavirus: the Italian security residences put to the test in a public health emergency. Psychiatry and Clinical Neurosciences. 